\title{
Principles to use reverse osmosis for drinking water supply and reduce concentrate disposal
}

\author{
Alexei Pervov", Dmitry Spitsov, and Alexei Saimullov \\ Moscow State University of Civil Engineering, Yaroslavskoe shosse, 26, Moscow, 129337, Russia
}

\begin{abstract}
Possibilities to reduce reverse osmosis concentrate flow and operational costs were investigated. The existing solutions are described using chemical softening and evaporation techniques. The presented article is devoted to investigation of possibilities to remove calcium carbonate from concentrate due to calcium deposition on "seed" crystals. A new technique to reduce concentrate flow is presented that consists of operation of membrane unit in circulation mode whereby concentrate is circulated through a "seed" reactor. In reactor calcium and carbonate ions deposit on the "seed" crystals thus reducing scaling hazard and concentrate TDS value. Water treatment flow diagram is presented and technical parameters of membrane facilities are determined that enables us to design the whole membrane process. Product flow on each membrane stage is determined to calculate the required amounts of membrane modules on each stage; scaling rates and calcium carbonate deposition rates are evaluated; caustic consumption is determined. Experimental technique is proposed and described to determine scaling rates in membrane modules and the amount of hardness removed from concentrate after treatment by the "seed" and the minimal volume of concentrate that could be reached. Relationships are presented to determine operational costs (energy and chemicals consumption), scaling rates in membrane modules on the first and second stages and total hardness removal during concentrate recirculation through the "seed" reactor on the third stage.
\end{abstract}

\section{Introduction}

Attempts to decrease concentrate flow were undertaken using ion-exchange to remove calcium from the feed water during pretreatment $[8,9]$ and physical and chemical methods $[10,11]$. Also attempts were made to deposit calcium from concentrate to further decrease its flow $[10,11]$.

To successfully implement complete reverse osmosis concentrate utilization, a "Zero Liquid Discharge" (ZLD) method is developed that uses chemical softening in pellet reactors [11]. To increase calcium carbonate supersaturation rates, degasifies are used prior to reactors to remove carbon dioxide from concentrate [13].

Meanwhile, the use of antiscalants, even the most efficient antiscalants $[14,15]$, demonstrates limited abilities to prevent scaling and reduce concentrate flow when ground

*Corresponding author: ale-pervov@yandex.ru 
water with high hardness is purified [16 - 18]. A numbers of publications [1, 11, 13, $18-$ 20] describe application of "seed" crystals added to concentrate to remove "excessive" calcium. Deposition of calcium due to supersaturation reached during water transport through reverse osmosis membrane was implemented using tubular membranes and addition of calcium sulphate "seeds" into circulating concentrate [13].

Authors of a number of papers [18 - 20] has attempted to use a specially designed "open-channel" reverse osmosis modules and dosing of "seed" crystals [19] in the feed water to initiate crystal growth in membrane channels. As it was already mentioned in [20], such an approach demonstrated a main disadvantage that consisted of sedimentation of crystals on membrane surface. Present work is aimed at experimental testing of a new simplified technique that provides calcium crystallization in a specially designed sedimentation reactor, to radically reduce concentrate volume and to remove concentrate together with the sedimented deposits. Main equations and experimental dependencies that describe deposition rates of calcium carbonate and magnesium hydroxide on the "seed" crystals depending on recovery and "seed" amount values are obtained.

It was concluded in [20] that calcium carbonate is deposited on the "seed" crystals and chemicals (caustic) are used only to produce "seeds" that constitute less than $2-3$ per cent of total stoichmetric amount of all removed hardness. The main advantage of the new developed technique is that hardness removal occurs without the use of chemicals and the driving force of crystallization process is attributed to increase of calcium and bicarbonate ions in reverse osmosis concentrate [20]. Also calcium carbonate depositions on the «seed» crystals decreases concentrate TDS that facilitates its further concentration by reverse osmosis facilities and recovery increase. Present work was aimed at development of this new concept of reverse osmosis application for drinking water supply without a problem of concentrate disposal. It is well known, that concentrate handling is one of the most important unsolved problems that slow down wide successful application of reverse osmosis techniques in drinking water supply practice despite its obvious efficiency.

As it is well known, addition of antiscalants does not completely eliminate scaling on membranes but only reduces rates of its formation [14 - 16]. At high recoveries during reverse osmosis facilities operation concentration values of calcium and bicarbonate ions are very high and antiscaling efficiencies of the majority of commercial antiscalants is limited. Therefore, concentrate flow rate of the majority of reverse osmosis facilities that treat ground water is maintained not less than $25-30$ per cent of the feed water flow that enters membrane facility $[2,3,17]$. Concentrate discharge into the drain increases price of the purified water as the customer pays not only for purified water but for the discharged wastewater as well. Also, location of water treatment facility can influence concentrate disposal problem: often water treatment facilities are located near the intake and do not provide conditions for concentrate discharge. In these cases concentrate flow can be reduced to minimum value to enable us to take out concentrate by road to deliver to the nearest wastewater treatment facilities. Examples of such approach are demonstrated by a number of municipalities in California that use reverse osmosis techniques to produce drinking quality water from well water $[1,2]$. To reach high recoveries, concentrate is softened in pellet reactors and further concentrated by a second reverse osmosis stage [1]. Similar approach is used in a number of drinking water supply projects in Moscow vicinity. In our projects authors have developed a new technique that uses seed crystals in reactor. Reverse osmosis system is operated in circulation mode and concentrate passes through reactor tank with seed crystals that grow due to supersaturation reached after treatment of concentrate in reverse osmosis module.

Main goals of the conducted experimental programs were:

1. Determination of scaling rate values at the each stage of membrane treatment;

2. Determination of the "seed" crystals growth in reactor in circulation mode; 
3. Determination of different ion concentrations values as a function of volume reduction coefficient $\mathrm{K}$ values;

4. Evaluation of membrane flux reduction with coefficient $\mathrm{K}$ increase;

5. To develop a method to determine values of design parameters such as: daily amount of concentrate; specific energy consumption; concentrate accumulation tank volume; amounts of membrane modules on each stage.

\section{Experimental program conductance}

Experiments were conducted using Moscow tap water. Membrane elements of 1812 standard were used tailored with low pressure reverse osmosis BLN membranes and nanofiltration 70 NE membranes supplied by CSM Company (Korea). "Seed" crystals were produced by addition of caustic (sodium hydroxide) to reverse osmosis concentrate. Pump RO 900-220 model was used Supplied by Raifil Company (Russia). "Aminat-K" antiscalant supplied by "Ecos-1" Company (Russia) was added to the feed water. "Aminat-K" is widely used in reverse osmosis application practice commercial antiscalant. This is a mixture of nitrilitris (methylenephosphonic) and methyliminobis (methylenphosphonic) acids. Antiscalant doses were 1 and 5 ppm.

Concentrations of calcium and magnesium ions were evaluated using EDTA titration. TDS values were evaluated using electric conductivity method. "Seed" crystals were produced by addition of 300 milliequivalents of $1-\mathrm{N}$ sodium hydroxide (caustic) solution to 10 liters of the feed water that is a stoichmetric amount to completely remove hardness from 10 liters of reverse osmosis concentrate. A flow diagram of the test membrane unit is shown on Figure 1.

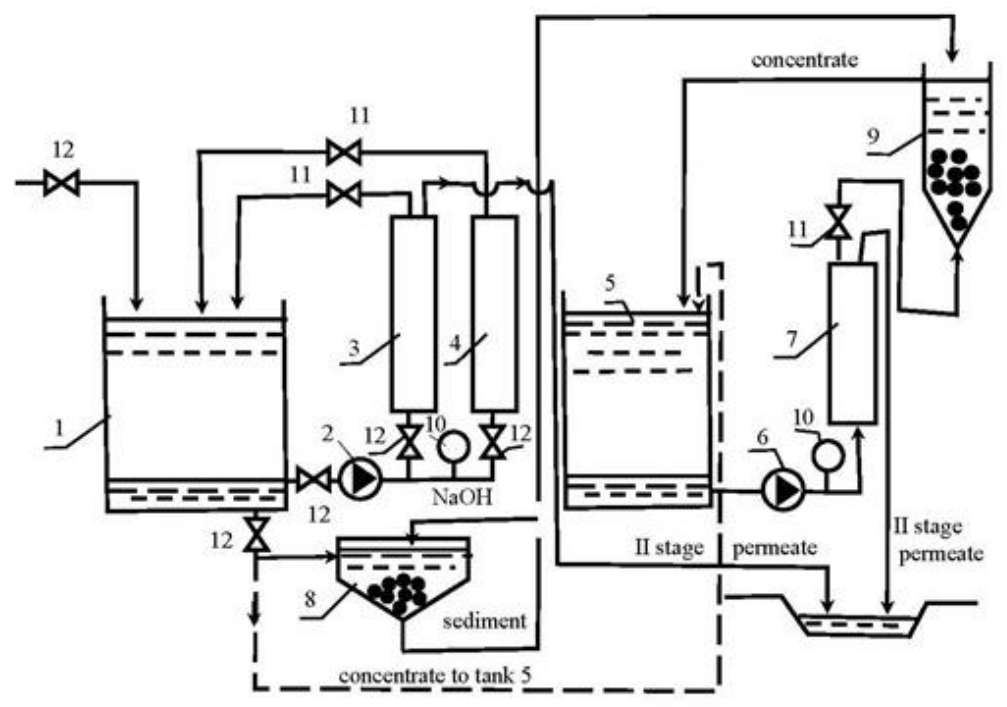

Fig. 1. A flow diagram of the test membrane unit and principles of experiment conductance: 1 - feed water tank; 2 - first stage pump; 3 - first stage reverse osmosis membrane module with BLN membranes; 4 - first stage membrane nanofiltation module with $70 \mathrm{NE}$ membranes; 5 - second stage concentrate accumulation tank; 6 - third stage pump; 7 - third stage nanofiltration membrane module; 8 - calcium carbonate and magnesium hydroxide deposition and sedimentation tank; 9 sedimentation reactor/tank; 10 - manometer; 11 - pressure regulation valve; 12 - ball valves. 
The feed water was added to tank 1 and delivered by the pump 2 into membrane element 3 with reverse osmosis membranes. The concentrate volume was reduced to 20 liters that corresponds to coefficient $\mathrm{K}$ value of 5 . After concentrate volume was reduced to 10 liters volume, a stoichmetric amount of caustic solution was added to the tank 1 to deposit hardness. The deposited and sedimented crystals were added to reactor tank 8 . During operation of the first stage membrane module in circulation mode, values of $\mathrm{K}$ coefficient veer determined as a ratio of feed water volume in feed tank 1 to the volume at different moments of the experiment. After concentrate volume reached 20 liters, the reverse osmosis module 3 was turned off with the help of ball valves 12 and switched to the nanofiltration module 4 and the experiment was repeated and concentrate volume in tank 1 was reduced to 10 liters. This obtained concentrate volume was added to tank 5 . From tank 1 the concentrate was pumped into the third stage module 7 . The third stage concentrate after membrane module 7 was pumped into reactor 8 and after passing through reactor returned to the tank 5. After concentrate contact with sediment calcium deposited on the "seed" crystals. After concentrate returned to tank 5 the cycle repeated. While the third stage permeates from module 7 discharged the concentrate volume in tank 5 reduced and concentrations of different ions in concentrate increased. Calcium and magnesium ions deposited on the "seed" crystals and calcium concentration reduced by the equivalent amount of deposited calcium carbonate. Chemical composition of the feed water, second stage concentrate and concentrate after contact with "seed" crystals are shown in Table 1.

Table 1. Chemical composition of the feed water, second stage concentrate and concentrate after treatment in the "seed" reactor.

\begin{tabular}{|c|c|c|c|c|}
\hline & Characteristics & Feed water & $\begin{array}{c}\text { Concentrate, } \\
\mathrm{K}=10 \\
(70 \mathrm{NE} \text { membranes })\end{array}$ & $\begin{array}{c}\text { Concentrate (K=10) } \\
\text { After contact with } \\
\text { "seed" crystals } \\
(150 \text { meq/liter })\end{array}$ \\
\hline 1 & Hardness, meq / liter & 3.1 & 20.1 & 12.2 \\
\hline 2 & Calcium, meq / liter & 4.03 & 17.8 & 10.5 \\
\hline 3 & Magnezium, meq / liter & 0.93 & 43 & 1.7 \\
\hline 4 & Bicarbonate, meq / liter & 3.0 & 15.2 & 1410 \\
\hline 5 & TDS, ppm & 360 & 2010 & 9.3 \\
\hline
\end{tabular}

In the beginning of the test run reverse osmosis module with BLN membranes was used to reach coefficient $\mathrm{K}$ value of 4 (to reduce the initial feed water volume in tank 1 by 4 times). Then the volume in tank 1 was reduced by 2.5 times using nanofiltration membrane to reach the volume reduction coefficient $\mathrm{K}$ value of 10 . During operation of membrane modules on the first and on the second stages, calcium carbonate growth rates were determined. Scaling rates were determined in conformity with the method developed by the authors and described in previous publications [18, 21].

Results of scaling rates determination are presented on figures $2-4$. Figures 2(a) and 3(a) show dependencies of calcium concentration in concentrate and permeate on the volume reduction coefficient $\mathrm{K}$ values. The amount of calcium carbonate deposited in membrane channels was calculated by mass balance as a difference between calcium amount in the feed water and in concentrate in the end of the test run [21]. Comparison of scaling rates in reverse osmosis and nanofiltration modules are shown on figure 4. 
Experimental results of calcium carbonate growth on the "seed" crystals are presented on Figure 5.

Figure 5(a) demonstrates growth of calcium and magnesium concentration value depending on coefficient $\mathrm{K}$ value. For comparison dependencies of calcium and magnesium concentration values on $\mathrm{K}$ value in experiment with "seed" and without "seed" crystals addition. The dotting line shows predicted concentration values as coefficient $\mathrm{K}$ value during test run without "seed" addition did not exceed the value of 4.Figure 5(b) shows dependency of concentrate TDS value calculated with account for removed calcium carbonate on $\mathrm{K}$.

\section{Discussions of the results}

Figures 2 and 3 demonstrate results of "Aminat-K" testing and its behaviour at the first and the second stages (Figure 1). As it is shown on Figures 2 and 3, both $1 \mathrm{ppm}$ and $5 \mathrm{ppm}$ antiscalant doses provide similar efficiencies and ensure safe operation of membrane facility.

As it is can be seen on Figure 2, treatment of feed water using reverse osmosis membranes and subsequent reduction of concentrate flow to a value of 10 per cent of the feed water flow (that corresponds to coefficient $\mathrm{K}$ value of 10) indicates that calcium carbonate scaling rate in the second stage modules is even lower than in the modules at the first stage (Figure 2). This provides sufficient economical advantages of the use of nanofiltration membranes due to low energy and reagent consumption.

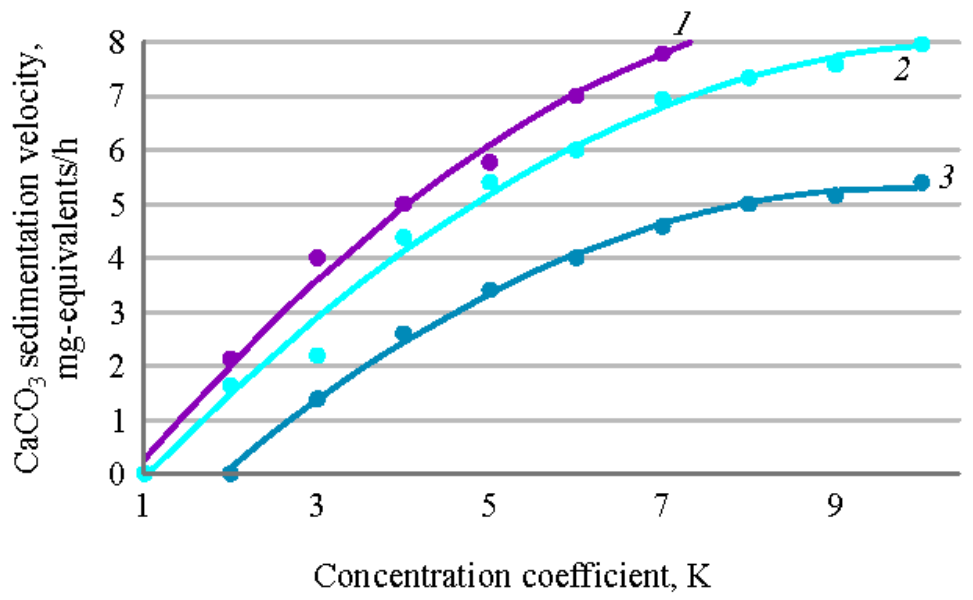

Fig. 2. Comparison of scaling rates in nanofiltration and reverse osmosis membrane modules dependencies of scaling rate values on K: 1 - reverse osmosis BLN membranes, "Aminat-K" dose 5 ppm; 2 - nanofiltration 70 NE membranes, "Aminat-K" dose is 1 ppm; 3 - nanofiltration membranes without antiscalant addition.

Experiments to deposit calcium carbonate and magnesium hydroxide on the "seed" crystals were conducted using antiscalant dose of $5 \mathrm{ppm}$ that is a standard dose used in ground water treatment with reverse osmosis facilities. The presence of antiscalants in concentrate prevents calcium carbonate scaling [21], but it was demonstrated that at high coefficient $\mathrm{K}$ values and high calcium and bicarbonate ion concentration values crystal growth is continued even in the presence of antiscalants [21]. As it can be seen on Figure 3, calcium carbonate and magnesium hydroxide are deposited on the "seed" crystals. The 
growth of magnesium hydroxide can be explained by formation of magnesium hydroxide crystals after stoichmetric addition of caustic to concentrate to remove total hardness [21]. Figure 3(a) shows experimental curves of calcium and magnesium concentrations versus $\mathrm{K}$ in experiments with and without "seed" crystals addition. The growth of calcium carbonate on the "seed" reduce concentrations of calcium and bicarbonate ions and TDS of concentrate (Figure 3,b). The calculated amounts of deposited calcium carbonate and magnesium hydroxide are presented on Figure 3 (c). Figure 6 demonstrates results of experimental program to determine main operational parameters of concentrate flow reduction measures: a) - dependencies of calcium and magnesium concentration on $\mathrm{K}$ values on each membrane stage; $b$ ) reduction of specific membrane flux with coefficient $\mathrm{K}$ value growth.

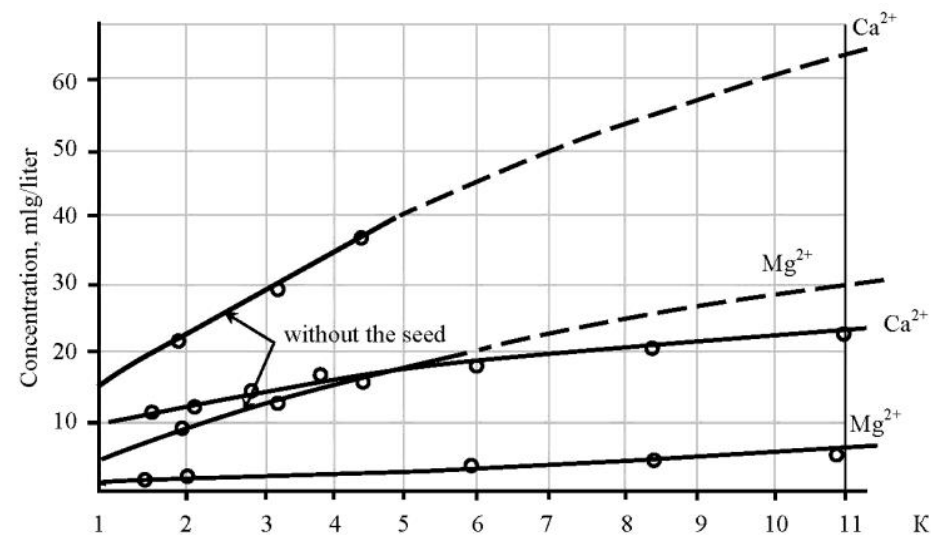

a)

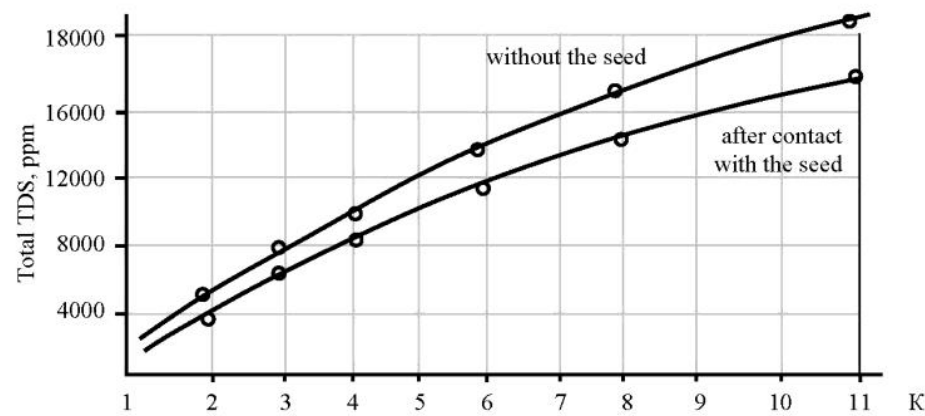

b)

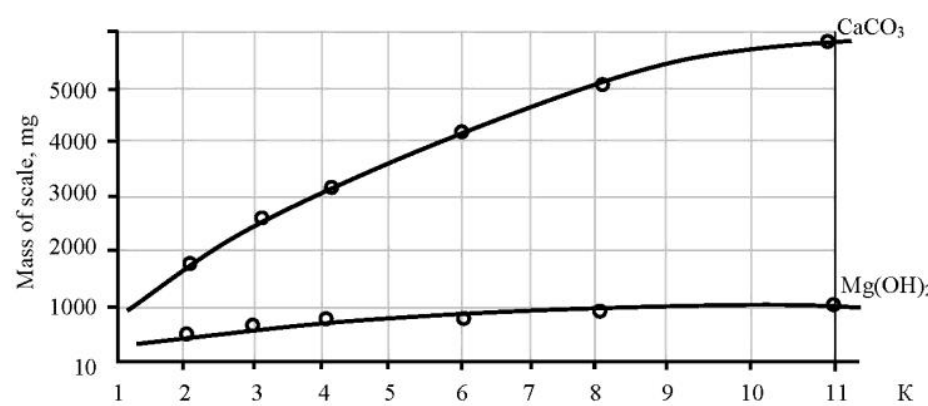

c)

Fig. 3. Growth of calcium carbonate and magnesium hydroxide depending on coefficient $\mathrm{K}$ values: a) Calcium and magnesium concentrations versus $\mathrm{K}$; b) TDS values of circulating concentrate versus $\mathrm{K}$; c) amounts of deposited calcium carbonate and magnesium hydroxide versus $\mathrm{K}$. 


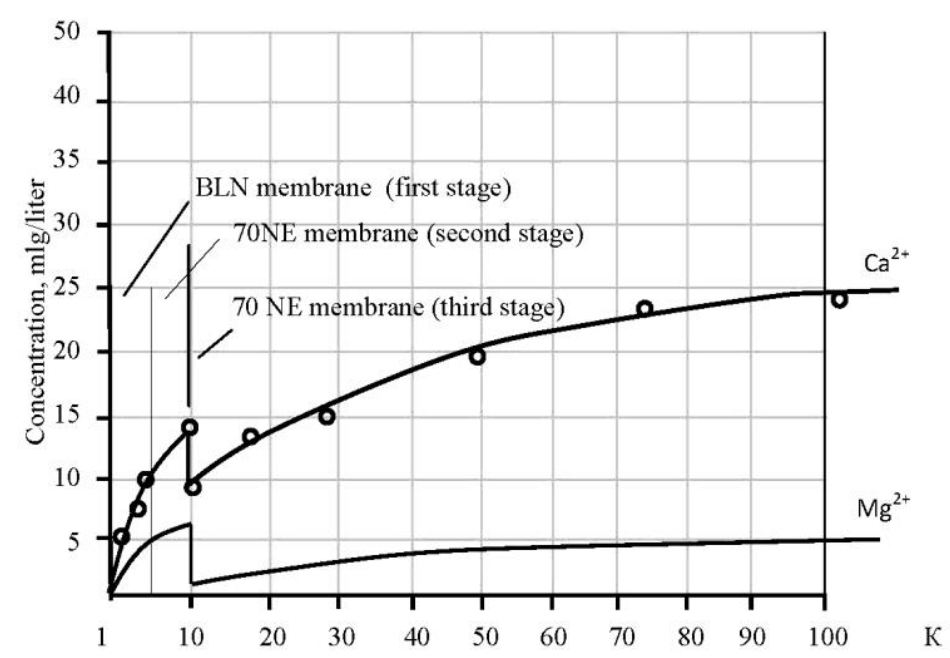

a)

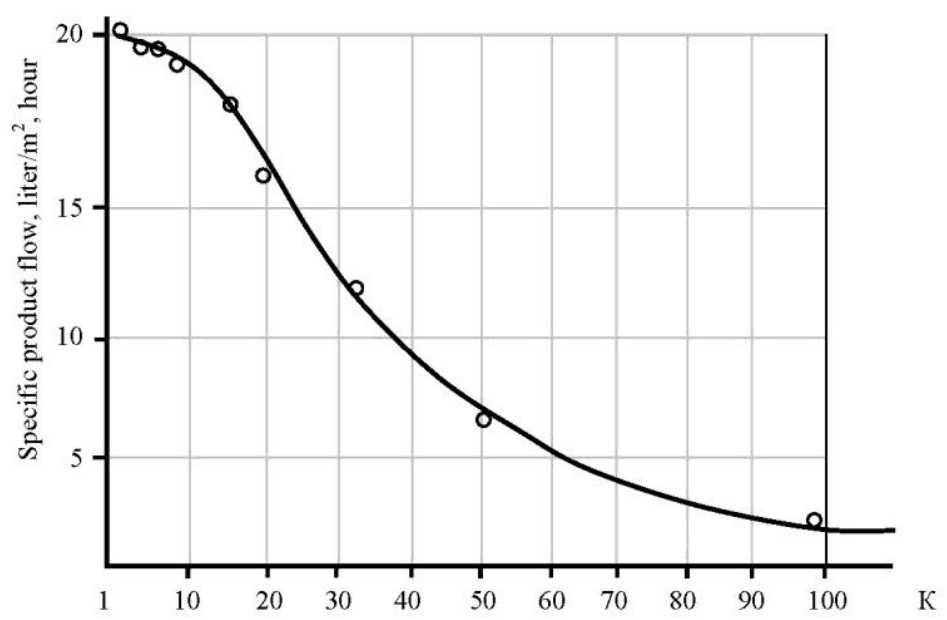

b)

Fig. 4. Experimental results of determination of main operational parameters of seed growth membrane reactor: a) dependencies of calcium and magnesium concentrations on coefficient $\mathrm{K}$ value in concentrate on the third stage; $b$ ) reduction of specific membrane flux with coefficient $\mathrm{K}$ value growth.

\section{Industrial application of the results}

The described technique was used in the project to supply quality drinking water for municipality in Vidnoe town in Moscow Region. The ground water contained excessive hardness, ammonia and fluoride. Product water capacity was 1000 cubic meters per day and designed concentrate flow value constituted 5 cubic meters or less [20]. The concept of concentrate utilization consists of its treatment in sedimentation tank / reactor (Figure 5) where all salts dissolved in the feed water are accumulated in reactor due to operation of membrane unit in circulation mode. The contents of the tank are updated once a day (24 
hours). Principles of the developed techniques to reduce concentrate flow are shown on Figure 5.

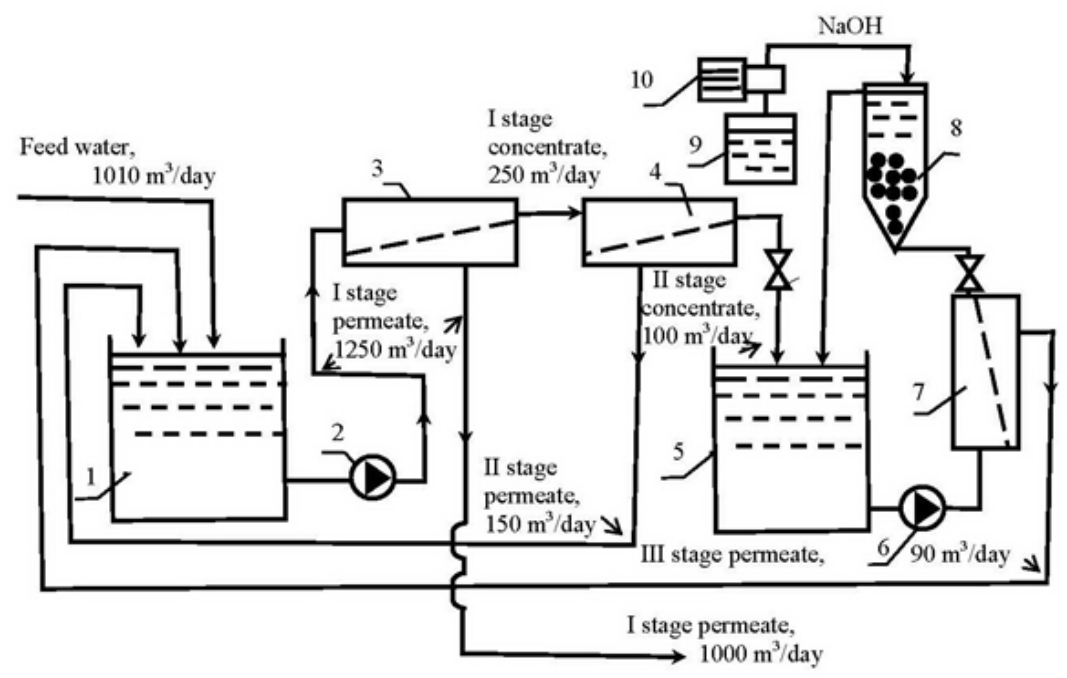

Fig. 5. A flow diagram of ground water treatment to produce drinking water using reverse osmosis and nanofiltration membrane stages and concentrate flow reduction stage: 1 - feed water tank (to collect water after pretreatment, iron removal and antiscalant addition); 2 - first stage high pressure pump; 3 - first stage reverse osmosis; 4 - second stage nanofiltration membranes; 5 - second stage concentrate accumulation tank; 6 - second stage high pressure pump; 7 - third stage nanofiltration membrane for concentrate flow reduction; 8 - sedimentation tank / reactor with "seed" crystals; 9 caustic dosing tank; 10 - pressure regulation valve.

For practical application of the results we need to determine values of all parameters of all elements of flow diagram shown on Figure 5, such as: volumes of tanks, reagent consumption, number of membrane modules on each stage.

Caustic consumption to produce "seed" crystals does not exceed $2-2.5$ per cent of chemical amount required for chemical softening when caustic or lime are added in stoichmetric amounts [21]. A specially designed "open channel" membrane modules were used on the third stage demonstrate low scaling propensities and ensure safe reliable operation [19].

The volume of concentrate that is discharged from membrane facility (which is a sum of volumes of tank 5 and 8 ) is determined by solution of mass balance equations: the amount of salts collected in concentrate tank equals to the amount of salts in feed water and in permeate. The predicted concentration of TDS values and calcium concentration values growth with time in reactor tank 8 (Figure 5) are shown on Figure 6. Calculations were performed for the case of pilot plant with permeate product flow of 100 liters per hour and second stage concentrate flow entering reactor of 10 liters per hour. The plots account for the reduction of membrane product flow with $\mathrm{K}$ value growth (Figure 4,b). The second stage membrane flux equals to concentrate flow entering the tank 5 (Figure 5). The selection of cross-flow rate of the third stage membrane concentrate that further enters and passes through reactor should ensure upstream velocity to prevent removal of particles from the reactor 8 (Figure 5). The volume of the tank and reactor that contains concentrate can be calculates as daily feed water volume divided to maximum coefficient $\mathrm{K}$ value. Concentrate can be removed once a day. For example, for the reverse osmosis facility that produces 1000 cubic meters per day and coefficient $\mathrm{K}=200$ concentrate volumes by the end of daily operation have a value of 5 cubic meters to take it out by road. Maximum $\mathrm{K}$ value is 
selected according to maximum reasonable concentration of salts treated by $70 \mathrm{NE}$ membranes of $70000 \mathrm{ppm}$. Concentrate TDS can be calculated taking into account deposition of calcium carbonate and reduction of salinity.

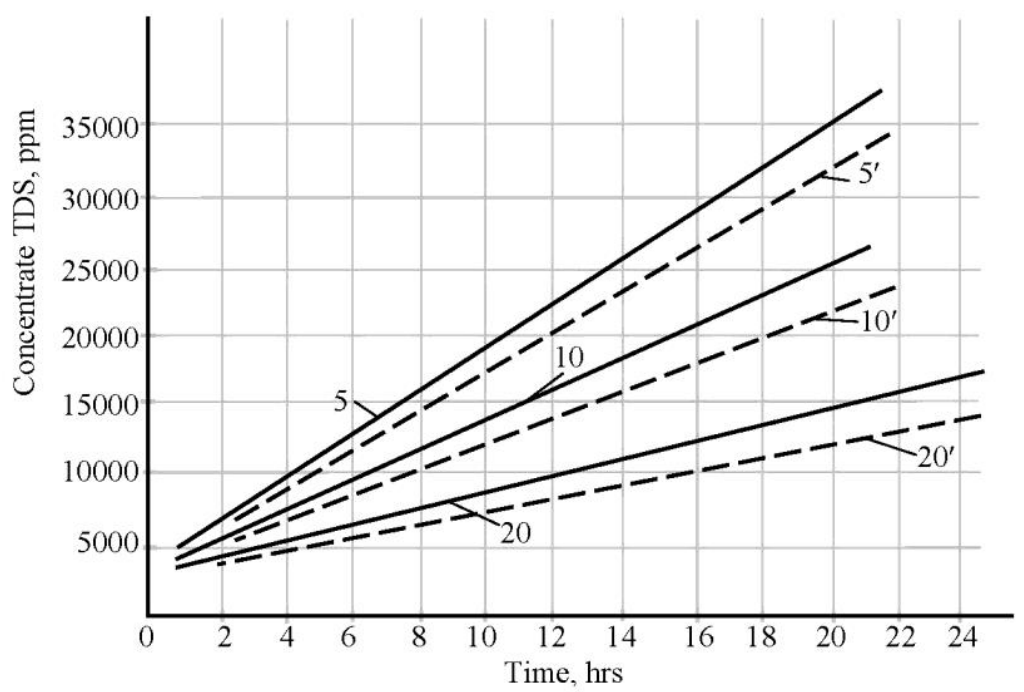

a)

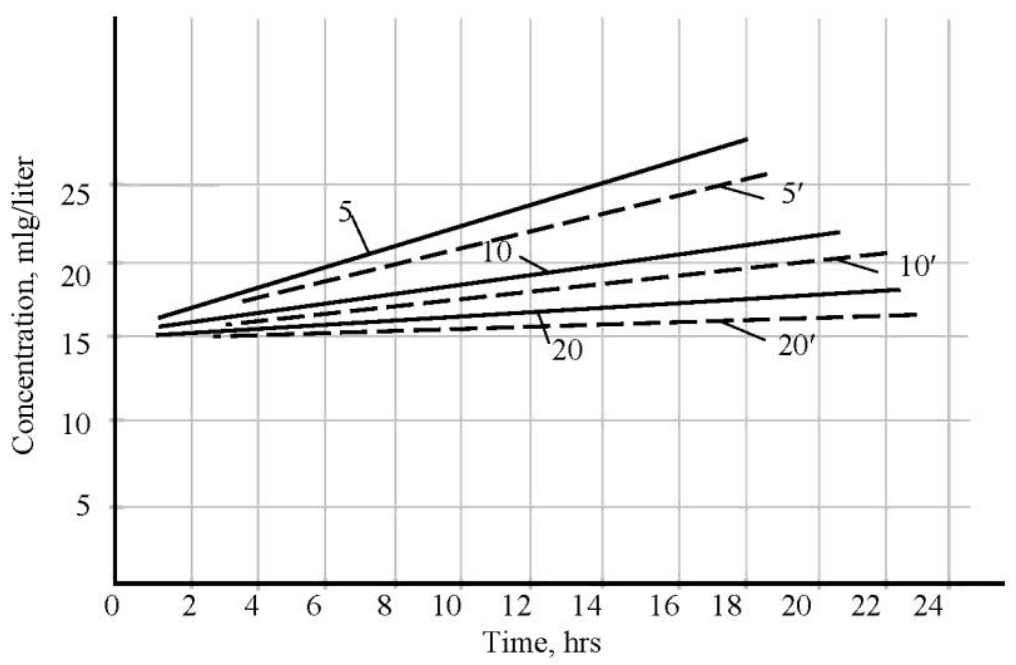

b)

Fig. 6. Growth of salt concentration values with time in reactor: a) the dependencies of TDS versus time calculated for various reactor tank volumes of 5, 10 and 20 liters; b) the dependencies of calcium concentration values on time for various reactor volumes of 5, 10 and 20 liters.

\section{Conclusions}

1. A new technique is developed to reduce concentrate flow rate to minimum possible value by its treatment in reactor with "seed" crystals;

2. Experiments demonstrated that calcium deposit on the "seed" crystals; 
3. Experiments demonstrated that not only calcium but magnesium as well is deposited on "seed" crystals;

4. Parameters of the process are experimentally evaluated. Process design is developed basing on maximum $\mathrm{K}$ value that is determined according to the maximum reached TDS value of $7000 \mathrm{ppm}$ in concentrate.

The authors would like to express gratitude to the Russian Foundation for Basic Research for the financial support for this project (RFBR grant № 19-08-00982A).

\section{References}

1. Yoram Cohen, Raphael Semiat, Anditya Rahardianto. A Perspective on Reverse Osmosis Water Desalination: Quest for Sustainability/ AIChE Journai, June 2017, Vol.3, No.6, DOI 10.1002/aic.15726

2. Arun Subramani, Joseph G. Jacangelo. Treatment technologies for reverse osmosis concentrate volume minimization: A review. Separation and Purification Technology, Volume 122, February 2014, Pages 472-489

3. Sung Hee Joo, Berrrin Tansel. Novel technologies for reverse osmosis concentrate treatment: A review. Journal of Environmental Management, Volume 150, 1 March 2015, Pages 322-335

4. Lee Foster, Jacqueline Kepke, Rebecca Sommer, Damian McCann. Hold the salt; Innovative Treatment of RO Concentrate. Proceedings of the WaterEnvironment Federation, 2008 (14): Pp. 219-230, DOI: 10.2175/19384708788734539

5. Turek M.,Mitko K., Dydo P., Laskovska E., JacobicKolon A. Prospects for high water recovery membrane desalination // Desalination. 2017. Vol. 401. Pp. 180-189. DOI: 10.101/j.desal.201.07.047

6. Jamaly S., N.N., Darwish N.N.,Ahmed I., Hasan S.W. A short review on reverse osmosis pretreatment technologies // Desalination. 2014. Vol. 354., Pp. 30-38. DOI: 10.1016/j.desal.2014.09.017

7. Goh P.S., Lau W.J., Othman M.H.D., Ismail A.F. Membrane fouling in desalination and its mitigation strategies // Desalination. 2018. Vol. 425. Pp. 130-155. DOI: 10.1016/j.desal.2017.10.018

8. Jiang S.,Li Y.,Ladewig B.P. A review of reverse osmosis membrane fouling and control strategies // Science of the total environment. 2017. Vol. 595. Pp. 567-583. DOI: $10.1016 /$ j.scitotenv.2017.03.235

9. Suratt W.B., Adrews D.R., Pujals V.J., Richards S.A. Design considerations for major membrane treatment facility for groundwater // Proceedings of the Conference on Membranes in Drinking and Industrial Water Production. 2000. Vol. 1. Pp. 61-70

10. Salman M.A., Al-Nuwaibit G., Safar M., Al-Mesri A. Performance of physical treatment method and different commercial antiscalants to control scaling deposition in desalination plant // Desalination. 2015. Vol. 369. Pp. 18-25. DOI: 10.1016/j.desal.2015.04.023

11. Veespareni S., Bond R. Getting this last drop: new technology for treatment of concentrate // Tianjin IDA World Congress 2013 on Desalination and Water Reuse, October 2025, China 2013, TIAN 13-357 
12. Li C. et al. Membrane fouling mitigation by coupling applied electric field in membrane system: Configuration, mechanism and performance // Electrochimica Acta. 2018. V. 287. Pp. 124-134

13. Shalid M.K., Pyo M., Choi Y-G. The operation of reverse osmosis system with $\mathrm{C} 02$ as a scale inhibitor: A study on operational behaviour and membrane morphology // Desalination. 2018. V. 426. P.p. 11-20

14. Chaussemier M., Pourmohtasham E., Gelus D., Pecoul N., Perrot H., IEGION j., Cheap-Charpentier H., Homer O. State of art of natural inhibitors of calcium carbonate scaling. A review article // Desalination, 2015, v. 356, pp. 47-55. DOI: 10.1016/jdesal.2014.10.014

15. Pramanik B.K., Gao Y., Fan L., Roddick F.A., Liu Z. Antiscaling effect of polyaspartic acid and its derivative for RO membranes used for saline wastewater and brackish water desalination // Desalination. 2017. Vol. 404. Pp. 224-229. DOI: 10.1016/ j.desal.2016.11.019

16. Li X., Hasson D., Shemer H. Flow conditions affecting the induction period of CaSO4 scaling on RO membranes // Desalination. 2018. Vol. 431. Pp. 119-125. DOI: 10.101/j.desal.2017.08.014

17. Zimmer K.,Hater W., Icart A. Jaworski J., Kruse N., Braun G. The performance of polycarboxylates as inhibitors for $\mathrm{CaCO}_{3}$ scaling in reverse osmosis plants // Desalination and Water Treatment. 2016. Vol. 57. Issue 48-49. Pp. 2312-23175. DOI: 10.1080/19443994.2015.1133874

18. V.A. Golovesov, A.G. Pervov and A.D. Smirnov. Investigation of scaling mechanism on reverse osmosis membranes using "fluorescent" antiscalant. IPICSE 2020. IOP Publishing. IOP Conf. Series: Materials Science and Engineering. 1030(2021) 012064. doi: 10.1088/1757899XL/1030/1/012064

19. Alexei Pervov. Precipitation of calcium carbonate in reverse osmosis retentate flow by means of seeded techniques - a tool to increase recovery. Desalination (2015) pp. 140151

20. A. Pervov, A. Andrianov. Deposition of calcium and magnesium from PO concentrate by means of seed crystallization and production of softened water for technical purposes. Desalination and water treatment, (2018) 1-9, pp. 1-9. www.deswater.com, doi: $10.5004 /$ dwt.2018/2

21. Golovesov V.A., Rudakova G.Ya., Pervov A.G., Spitsov D.V. The choice of membranes and reagents designed for membrane systems used to treat ground water. Vestnik MGSU [Monthly Journal on Construction and Architecture]. 2020; 15(11): 1493-1504. DOI: 10.22227/1997-0935.2020.11.1493-1504 